\title{
Repeated Hemorrhage after Repair of Orbital Floor Fracture with a Silicone Implant
}

\author{
Hirohiko Kakizaki $^{{ }^{*}}$, Akihiro Ichinose ${ }^{2}$, Yasuhiro Takahashi ${ }^{1}$, Nobutada Katori ${ }^{3}$, \\ Alejandra A. Valenzuela ${ }^{1}$, Masayoshi Iwaki ${ }^{1}$ \\ ${ }^{1}$ Department of Ophthalmology, Aichi Medical University, Nagakute, Japan \\ ${ }^{2}$ Department of Plastic Surgery, Kobe University, Kobe, Japan \\ ${ }^{3}$ Department of Oculoplastic and Orbital Surgery, Seirei Hamamatsu General Hospital, \\ Hamamatsu, Japan \\ E-mail: ${ }^{*}$ cosme@d1.dion.ne.jp \\ Received January 19, 2011; revised August 30, 2011; accepted September 16, 2011
}

\begin{abstract}
A 16-year-old girl was accidentally kicked in her right eye by her cheerleading teammate in an exercise. Upward gaze ability of her right eye was severely impaired and computed tomography (CT) showed a trapdoor fracture of the right orbital floor. After surgical exploration, a silicone implant was inserted. No bleeding was confirmed at this time. The next day, CT detected a hematoma on the right orbital floor. The hematoma was drained and meticulous cautery was used to control any potential bleeding. The same silicone implant was re-inserted. Irrespective of attempts to avoid hemorrhage, this occurred twice after the respective evacuations. During a fourth operation, we removed the silicone implant simultaneously with hematoma evacuation. No hematoma has occurred since, and the patient's ocular movement has dramatically improved to a normal binocular single vision field. When repeated hemorrhages occur after an orbital floor fracture repair with insertion of a silicone implant, removal of the implant is an effective strategy to resolve the hemorrhage.
\end{abstract}

Keywords: Trapdoor Fracture, Orbital Floor, Silicone Implant, Hematoma, Hemorrhage, Removal

\section{Introduction}

Orbital hemorrhage in relation to alloplastic implants after repair of a blowout fracture is an uncommon entity [1-5]. Although this has been mainly reported as a late complication [3,4], acute hemorrhage also occurs [1,2]; however, the relationship between hemorrhaging and alloplastic implants has not been clarified. We report repeated hemorrhage after repair of an orbital floor fracture using a silicone implant, and the hemorrhage was resolved after removal of the implant.

\section{Case Report}

A 16-year-old girl was accidentally kicked in her right eye by her cheerleading teammate in an exercise. She had diplopia in upgaze at the time. She had an ophthalmological examination on the following day of the injury. Although the globe was not impaired, the upward gaze of the right eye was severely restricted. A right floor trapdoor fracture was confirmed by computed tomography (CT) (Figure 1)

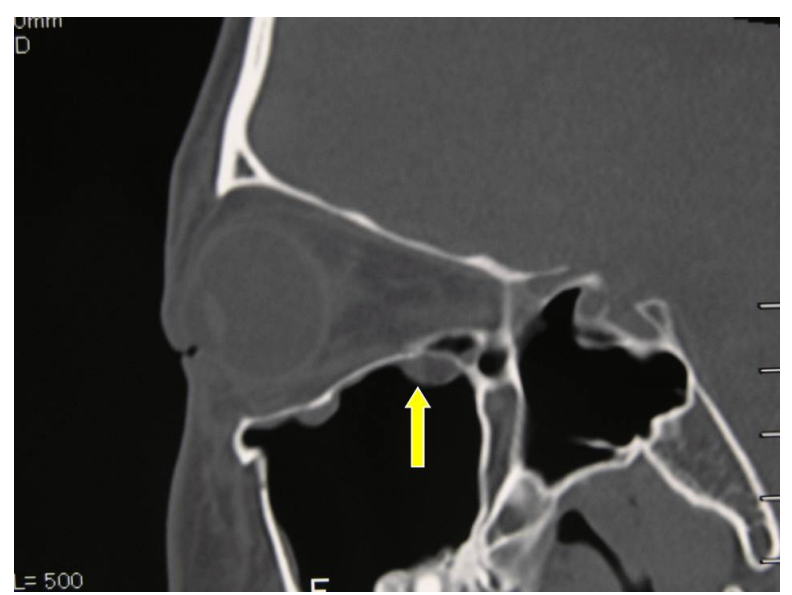

Figure 1. Sagittal computed tomography (CT) shows a trapdoor fracture of the right orbital floor (arrow). 
Surgical repair of the fracture was performed on the day of examinations via the swinging eyelid approach [6], releasing all the entrapped tissues followed by the insertion of a silicone implant on the floor to prevent prolapse of the orbital tissues. No hemorrhage was confirmed at the end of the operation.

On the following day of the operation, the patient developed right proptosis with normal optic nerve function. CT images demonstrated a hematoma on the right orbital floor. We evacuated the hematoma that day and the bleeding points were thoroughly cauterized. At the same time, the silicone implant was re-inserted and a drain was positioned between the implant and the floor.

The day after the second operation, no obvious hematoma was observed on CT (Figure 2(A)), and blood drainage was not evident on the second day after this operation. Three days later, however, right proptosis and severe chemosis developed (Figure 2(B)), and CT showed a large hematoma in the same location (Figure 2(C)). Although the hematoma had pushed the inferior rectus muscle towards the optic nerve, it did not induce any optic nerve dysfunction. Orbital exploration to remove the hematoma (Figure 2(D)) was carried out with meticulous cautery, and an additional two holes $3 \mathrm{~mm}$ in diameter each were made in the silicone implant to allow drainage of any orbital collection through the implant into the maxillary sinus.

On the day after the third operation, the patient had a hematoma again as shown by CT imaging. Although her right ocular movement was severely restricted, as her visual acuity and pupil reflex were not impaired, the hematoma was observed for 3 days with intravenous steroid therapy (dexamethasone $8 \mathrm{mg}, 4 \mathrm{mg}$, and $2 \mathrm{mg} / \mathrm{day}$, respectively). Since the symptoms were not resolved, we evacuated the hematoma again, and this time, the silicone implant was removed. There were no obvious bleeding points at any time. We did not explore the orbital contents to avoid further disturbance of the stable anatomy and to avoid the orbital tissues being entrapped again.
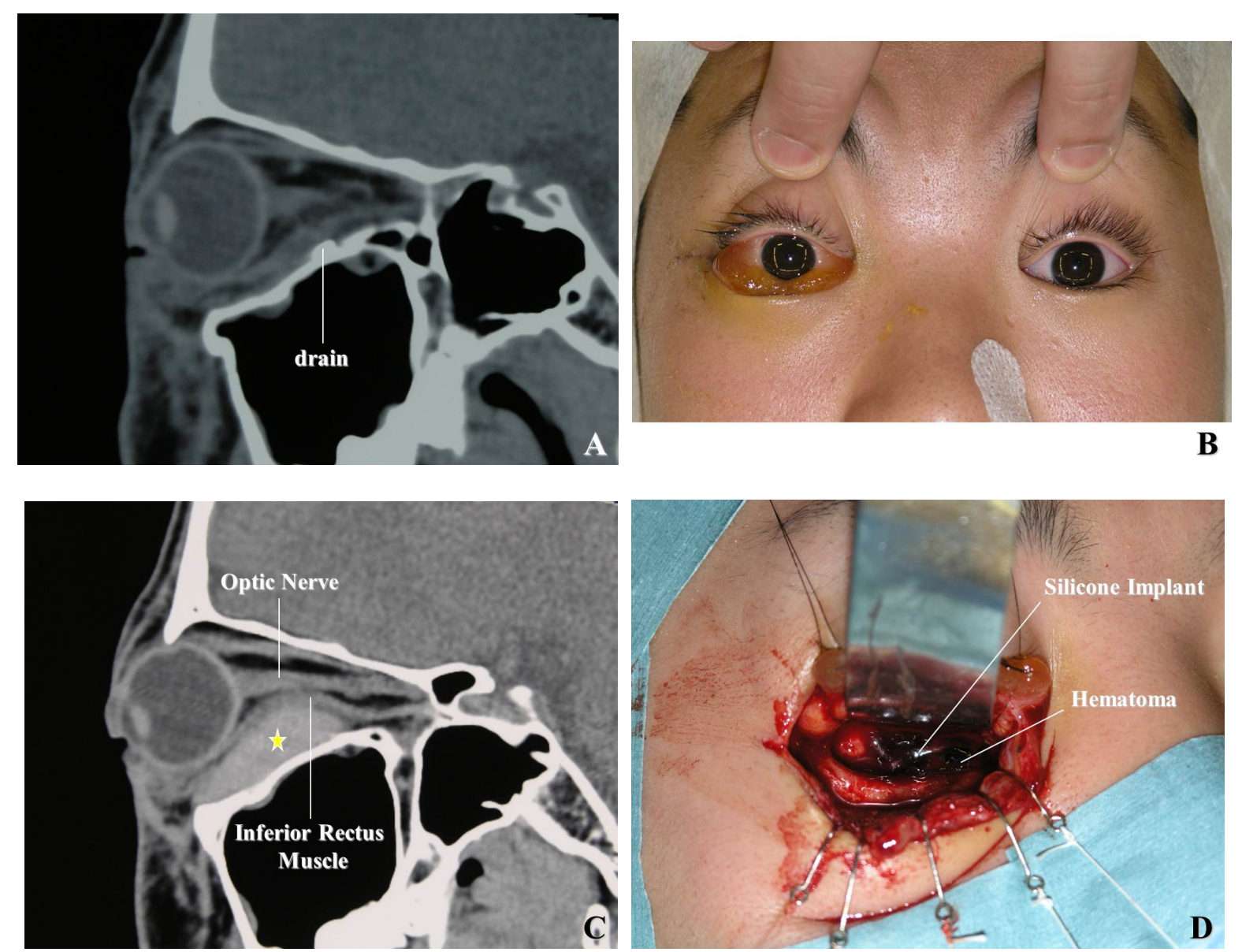

Figure 2. (A) Sagittal CT shows no hematoma on the day after the second operation; (B) Right proptosis and severe chemosis are shown 3 days after the second operation; (C) Sagittal CT shows a large volume of hematoma (asterisk) displacing the inferior rectus muscle and almost abutting the optic nerve; (D) The hematoma and silicone implant are shown during the third surgical exploration. 
After the fourth operation, no proptosis or chemosis were demonstrated (Figure 3), and 1 month later, the patient showed a full range of ocular movements and a normal binocular single vision field.

\section{Discussion}

Orbital hemorrhage is a frustrating complication after repair of a blowout fracture and the hemorrhage in relation to alloplastic implants has been mainly described as a late complication of repair [3-5]. The current report presented the possibility that an acute hemorrhage is also able to be induced by a silicone implant.

The cause of acute hemorrhage by a silicone implant is unknown, but the mechanism may be attributed to insufficient fixation of the mobile implant causing continuous irritation and erosion of the surrounding soft tissue and vessels. This small amount of movement may be enhanced by its smooth surface and by the overlying extraorbital muscles. This may explain the resolution of the hematoma after removing the implant.

Several studies recommend not to use a silicone implant because of its complications [5-9], but some other studies have reported a positive opinion for use of a silicone implant [10,11]. Therefore, silicone implant use is controversial. Although we experienced orbital hemorrhage caused by a silicone implant, other implant materials can also cause the same complication [5]. However, porous polyethylene implants are reported to be highly biocompatible, stable and durable for reconstruction of orbital defects with few complications [12,13]. Although use of this implant material may have prevented the hemorrhages, we could not use this material because of an administrative matter.

An implant with a textured surface has less contracture than that with a smooth surface [14]. Therefore, textured type implants are thought to be more biocompatible with

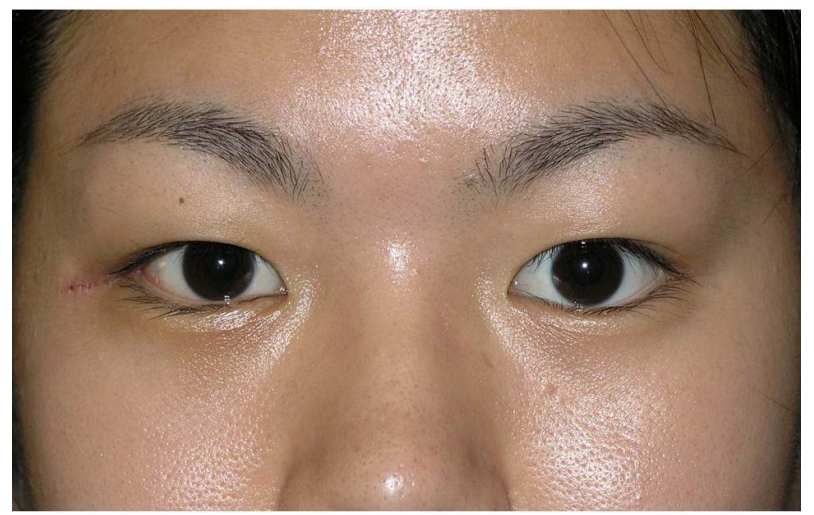

Figure 3. Proptosis and chemosis are completely resolved 1 week after the final operation. surrounding tissues. This is similar to porous polyethylene implants with a high biocompatibility $[12,13]$. If silicone implants were processed to have a textured surface, their biocompatibility and stability could be increased, causing less bleeding.

When there is an orbital hematoma, but visual function is not impaired, it may be best to perform evacuation of the hematoma a couple of days after the onset. At this time, the hemorrhage will have stopped and the orbital anatomy will become stable, which enables removal of the implant without collapsing the stable shape of the orbital content. This is an advantage to avoid further disruption of the stable anatomy and to avoid the orbital tissues being entrapped again against the fracture site.

In conclusion, when repeated hemorrhage after orbital floor fracture repair with a silicone implant occurs, removal of the implant is an effective strategy to resolve the hemorrhage.

\section{References}

[1] G. A. Wilcsek, M. Kazim, I. C. Francis, S Sharma, J. B. Reed and D. E. E. Holck. "Acute Orbital Hemorrhage," In: D. E. E. Holck and J. D. Hg, Eds., Evaluation and Treatment of Orbital Fractures-A Multidisciplinary Approach, Elsevier Saunders, Philadelphia, 2006, pp. 381389.

[2] C. W. Browning, “Alloplast Materials in Orbital Repair," American Journal of Ophthalmology, Vol. 63, No. 5, 1967, pp. 955-962.

[3] S. Warrier, V. C. Prabhakaran, G. Davis and D. Selva, "Delayed Complications of Silicone Implants Used in Orbital Fracture Repairs,” Orbit, Vol. 27, No. 3, 2008, pp. 147-151. doi:10.1080/01676830802077611

[4] J. A. Mauriello, J. C. Flanagan and R. G. Peyster, “An Unusual Late Complication of Orbital Floor Fracture Repair,” Ophthalmology, Vol. 91, No. 1, 1984, pp. 102107.

[5] J. S. Gilhotra, A. A. McNab, P. McKelvie and B. A. O’Donnell, "Late Orbital Haemorrhage around Alloplastic Orbital Floor Implants: A Case Series and Review," Clinical Experimental Ophthalmology, Vol. 30, No. 5, 2002, pp. 352-355. doi:10.1046/j.1442-9071.2002.t01-1-00555.x

[6] C. D. McCord Jr., “Orbital Decompression for Graves' Disease: Exposure through Lateral Canthal and Inferior Fornix Incision,” Ophthalmology, Vol. 88, No. 6, 1981, pp. 533-541.

[7] P. Cole, V. Boyd, S. Banerji and L. H. Hollier Jr., "Comprehensive Management of Orbital Fractures,” Plastic Reconstructive Surgery, Vol. 120, No. 7, Supplement 2, 2007, pp. 57S-63S. doi:10.1097/01.prs.0000260752.20481.b4

[8] J. A. Mauriello Jr., S. Hargrave, S. Yee, R. Mostafavi and R. Kapila, "Infection after Insertion of Alloplastic Orbital Floor Implants,” American Journal of Ophthalmology, 
Vol. 117, No. 2, 1994, pp. 246-252.

[9] D. R. Jordan, P. St Onge, R. L. Anderson, J. R. Patrinely and J. A. Nerad, "Complications Associated with Alloplastic Implants Used in Orbital Fracture Repair," Ophthalmology, Vol. 99, No. 10, 1992, pp. 1600-1608.

[10] S. J. Prowse, P. M. Hold, R. F. Gilmour, U. Pratap, E. Mah and F. W. Kimble, "Orbital Floor Reconstruction: A Case for Silicone. A 12 Year Experience,” Journal of Plastic, Reconstructive \& Aesthetic Surgery, Vol. 63, No. 7, 2010, pp. 1105-1109. doi:10.1016/j.bjps.2009.05.057

[11] J. C. Mwanza, D. K. Ngoy and D. L. Kayembe, "Reconstruction of Orbital Floor Blow-out Fractures with Silicone Implant," Bulletin Society Belge Ophtalmology, Vol. 280, 2001, pp. 57-61.
[12] J. J. Xu, L. Teng, X. L. Jin, Y. Ji, J. J. Li and B. Zhang, "Porous Polyethylene Implants in Orbital Blow-out Fractures and Enophthalmos Reconstruction," The Journal of Craniofacial Surgery, Vol. 20, No. 3, 2009, pp. 918-920. doi:10.1097/SCS.0b013e3181a2d728

[13] P. A. Rubin, J. R. Bilyk and J. W. Shore, "Orbital Reconstruction Using Porous Polyethylene Sheets,” Ophthalmology, Vol. 101, No. 10, 1994, pp. 1697-1708.

[14] G. P. Barnsley, L. J. Sigurdson and S. E. Barnsley. “Textured Surface Breast Implants in the Prevention of Capsular Contracture among Breast Augmentation Patients: A Meta-analysis of Randomized Controlled Trials," Plastic Reconstructive Surgery, Vol. 117, No. 7, 2006, pp. 2182-2190.

doi:10.1097/01.prs.0000218184.47372.d5 